\title{
WELCOMING ADDRESS BY THE CHAIRMAN OF THE SOC
}

\author{
Ladies and Gentlemen, Dear Colleagues,
}

It is with a feeling of great pleasure that I welcome you all on behalf of the Scientific Organizing Committee. We are delighted with the hospitality extended to us by the Elba International Physics Centre and the Univerisity of Pisa, and thank the chairman of the Local Organizing Committee, Prof.Dr. Federico Ferrini and his staff for their remarkable efforts.

Sponsored jointly by the International Astronomical Union and the Elba International Physics Center, this occasion is the sixth in the series of IAU symposia on hot massive stars, after No. 49 (Argentina 1971), No. 83 (Canada 1978), No. 99 (Mexico 1981), No. 116 (Greece 1985) and No.143 (Indonesia 1990). Those symposia, together with related colloquia and workshops on hot massive stars, have been the principle fora that have reviewed the status quo, witnessed the announcements of discoveries, and contributed to the stimulus for the future. This symposium will follow that tradition.

Wolf-Rayet binaries, their colliding winds and massive binary evolution are the main topics of the symposium. Recent observations and theoretical studies on the interaction between hot massive binary components have brought us more insight and more problems. Binary interaction can nowadays be observed over almost the entire electromagnetic spectrum, which should add to more complete models and constrain the number of free parameters in theories. Studies of the prototypes V444 Cygni and HD 193793 encourage us to observe all Wolf-Rayet binaries in much more detail, and each system will appear to be unique.

Seated here are 111 astronomers from 24 countries. You all have worked very hard to prepare for this week. I am confident that this symposium, with your valuable contributions and active participation, will serve its purpose and confront us all with thought-provoking ideas and stimulus for years to come.

Karel A. van der Hucht 